Revista Arbitrada Interdisciplinaria KOINONIA

Año V. Vol V. N9. Enero - Junio 2020

Hecho el depósito de Ley: FA2016000010

ISSN: 2542-3088

FUNDACIÓN KOINONIA (F.K). Santa Ana de Coro. Venezuela.

Byron Solorzano-Castillo; Jairo Castillo-Calderón; Renato Ambuludí-Hualpa

http://dx.doi.org/10.35381/r.k.v5i9.645

\title{
Aplicación para el diseño paramétrico de un prototipo de bomba de pistón alternativa y sumergible
}

\section{Application for the parametric design of an alternative and submersible piston pump prototype}

\author{
Byron Solorzano-Castillo \\ byron.solorzano@unl.edu.ec \\ Universidad Nacional de Loja, Loja \\ Ecuador \\ https://orcid.org/0000-0002-0071-2249 \\ Jairo Castillo-Calderón \\ idcastilloc@unl.edu.ec \\ Universidad Nacional de Loja, Loja \\ Ecuador \\ https://orcid.org/0000-0002-5321-4518 \\ Renato Ambuludí-Hualpa \\ arambuludih@unl.edu.ec \\ Universidad Nacional de Loja, Loja \\ Ecuador \\ https://orcid.org/0000-0002-9299-6022
}

Recibido: 15 de noviembre de 2019

Revisado: 18 de noviembre de 2019

Aprobado: 21 de diciembre de 2019

Publicado: 20 de enero de 2020

\section{RESUMEN}

La presente investigación trata sobre el desarrollo de una aplicación de software orientada al diseño mecánico parametrizado de un prototipo de bomba de pistón alternativa y sumergible, mediante la vinculación de herramientas informáticas de cálculo y CAD paramétrico. La metodología parte de la identificación del objeto de estudio, su descomposición en sistemas, partes y piezas que se grafican en software CAD (Inventor ${ }^{\circledR}$ ) con el nivel de detalle geométrico pertinente. Mediante el método de grafos dicromáticos (MGD) se plantea y resuelve el Modelo Matemático Integral (MMI) que describe funcional, estructural y geométricamente la bomba de pistón; se establece como variables de entrada el caudal y la altura de trabajo. El prototipo concebido está fundamentado en un estudio ingenieril que involucra a más de 


\section{Byron Solorzano-Castillo; Jairo Castillo-Calderón; Renato Ambuludí-Hualpa}

ecuaciones y funciones matemáticas, una serie de algoritmos basados en la tabulación de propiedades y variantes de materiales, normas, catálogos de partes y piezas comerciales.

Descriptores: Ingeniería de la producción; máquina herramienta; tecnología de materiales; tecnología de materiales; matemática combinatoria. (Palabras tomadas del Tesauro UNESCO).

\section{ABSTRACT}

This research is about the development of a software application oriented to the parameterized mechanical design of an alternative and submersible piston pump prototype, by linking computational calculation tools and parametric CAD. The methodology is based on the object of study identification; that is, its decomposition into systems, parts and pieces that are plotted in CAD software (Inventor ${ }^{\circledR}$ ) with the relevant level of geometric detail. The Integral Mathematical Model (IMM), which describes the piston pump functionally, structurally and geometrically, is proposed and solved using the dichromatic graph method (DGM). The flow rate and working height are established as input variables. The prototype conceived is based on an engineering study that involves a series of algorithms based on the tabulation of properties and variants of materials, standards, catalogs of parts and commercial parts more than equations and mathematical functions.

Descriptors: Production engineering; machine tools; materials engineering; combinatorial mathematics. (Words taken from the UNESCO Thesaurus).

\section{INTRODUCCIÓN}

Los países industrializados han desarrollado desde hace ya varios años herramientas de análisis y solución de problemas de diseño mecánico con la capacidad de vincularse entre sí para agilizar la obtención de resultados. Lo mencionado se logra por medio de modelos matemáticos con altos niveles de abstracción del objeto de estudio, ajustando los parámetros de diseño que posibilitan una construcción eficiente y de calidad (Marrero, 2009).

En nuestra región el proceso de diseño de máquinas y piezas es ineficiente debido a que aún se lo realiza de manera tradicional; es decir, se ejecutan cálculos de rutina innecesarios y en la mayoría de casos no se involucra herramientas de diseño computacionales (CAD), menos aún, una estructura de modelos matemáticos metódicamente organizados. En otros países, los diseños se desarrollan usando 
diversos softwares, con el fin de obtener un prototipo virtual, con base en componentes estandarizados, lo cual permite verificar y/o corregir su diseño, ahorrando tiempo y reduciendo costos.

El diseño paramétrico se centra en la resolución del problema de asignar la mejor combinación de valores posible a todos los parámetros necesarios para describir el objeto de diseño (Wielinga, Akkermans, \& Schreiber, 1995), en otras palabras, dado un problema informal de diseño, plantear y resolver un problema de cómputo que determinará los mejores valores de los parámetros del sistema (Marrero \& Martínez, 2008).

Existen herramientas CAD que ayudan al diseño de piezas mecánicas a través de bocetos y objetos 3D parametrizados. Además, cuentan con interfaz de programación que permiten vincular los parámetros del objeto de estudio con modelos matemáticos de diversa índole. Sin embargo, su uso puede resultar complejo y en ocasiones no es parte de la formación académica y profesional del diseñador.

Mediante el desarrollo de esta aplicación, que emplea una metodología viable, se pretende crear una referencia de la automatización del diseño mecánico de cualquier máquina o pieza.

El prototipo de bomba de pistón, alternativa y sumergible seleccionado, fue validado como se muestra en (Pineda, 2014). De forma general, este tipo de bombas tiene un amplio espectro de aplicaciones, tanto en el sector industrial como en energías alternativas, y se espera que, para futuros trabajos en estos campos de estudio, la aplicación y metodología derivadas de la presente investigación sean un aporte significativo.

En la interfaz de la aplicación desarrollada en Microsoft Excel®, basta con introducir los parámetros fundamentales de selección de sistemas de bombeo: caudal, entre 0 y 1,25 $\mathrm{l} / \mathrm{s}$, y cabezal de descarga, entre 0 a $100 \mathrm{mca}$, para obtener automáticamente el modelo virtual de la bomba en Autodesk Inventor ${ }^{\circledR}$. 


\section{Importancia del Problema}

La presente investigación constituye un aporte al valor práctico de la resolución de problemas de cómputo basado en el Método de los Grafos Dicromáticos y su integraciónal prototipado virtual paramétrico. De esto, resulta una aplicación que posibilita el análisis y la síntesis dentro del diseño de máquinas contribuyendo al desarrollo científico y tecnológico.

\section{METODOLOGÍA}

\section{Descripción y caracterización de la bomba alternativa y sus componentes}

La bomba seleccionada es de pistón, sumergible, de alto caudal, para la extracción de agua de pozos someros, cuyas especificaciones más relevantes se citan en la Tabla 1. Un esquema general de la bomba realizado en el software AutoCAD, con sus partes enumeradas se aprecia en el Gráfico 1.

\section{Planteamiento del modelo matemático}

Esquematización de los componentes de la bomba para generación de parámetros. En primera instancia se bosqueja en el software AutoCAD los componentes de la bomba en $2 \mathrm{D}$, sin considerar detalles como chaflanes, empalmes, ajustes y acabados superficiales. Posteriormente, se grafica los componentes en 3D en el software Inventor ${ }^{\circledR}$. Para ejemplificar lo mencionado, a continuación, se presenta el desarrollo para una de las piezas de la bomba, en este caso la denominada «brida inferior». El Gráfico 2 muestra la vista frontal del plano en dos dimensiones y el Gráfico 3 exhibe el sólido generado junto a los 22 parámetros geométricos propios de la pieza, estos últimos concebidos de forma automática por el software CAD 3D. Se procede de manera similar con todos los componentes del conjunto, obteniendo su modelo 3D. ensamblado, como se muestra en el Gráfico 4.

\section{Descripción del modelo matemático}

El MMI contempla 90 relaciones y 140 variables, divido en cuatro submodelos: geométrico, de resistencia de materiales, hidráulico y especiales; además, se contempla el uso de parámetros de diseño. El modelado geométrico de la bomba se 
Byron Solorzano-Castillo; Jairo Castillo-Calderón; Renato Ambuludí-Hualpa

basa en el prototipo desarrollado por (Pineda, 2014). En lo que respecta a la resistencia de materiales se considera el tipo de solicitación mecánica al que está sometido cada componente correspondiente al funcionamiento del conjunto. Por otro lado, la parte hidráulica involucra ecuaciones relacionadas con la presión y caudal de bombeo. Las funciones especiales son algoritmos provenientes de la tabulación de propiedades y variantes de materiales, normas, catálogos de partes y piezas comerciales del prototipo de bomba seleccionada.

Debido a los varios elementos que constituyen la bomba de pistón y las diversas relaciones que se derivan del modelado de cada uno de ellos, en este apartado se describe algunas funciones y parámetros de diseño utilizados esencialmente para modelar la brida inferior. Así, la Ecuación 1 es usada para el cálculo de su diámetro exterior con base en la norma ASME B16.5 (DNBrida, 2019).

$$
D_{\text {ext_brid_inf }}=D_{\text {int_brid_inf }}+6 \cdot D_{\text {pern_suj_brid }}
$$

Dónde:

$D_{\text {int_brid_inf }}$ : Diámetro interior de la brida inferior $(\mathrm{m})$

$D_{\text {pern_suj_brid }}$ : Diámetro del perno sujetador de la brida $(\mathrm{m})$

La Ecuación 2 establece la altura de la rosca para el perno del accesorio de entrada de agua, basada en recomendaciones de (Budynas, Keith, 2008); (Solórzano, 2014).

$$
\text { Prof }{ }_{\text {rosca_per_acc_entradH2O }}=0,8 \cdot D_{\text {per_acc_entradH2O }}
$$

Dónde:

$D_{\text {per_ace_entradH2o }}$ : Diámetro del perno del accesorio de entrada de agua $(\mathrm{m})$ 
Byron Solorzano-Castillo; Jairo Castillo-Calderón; Renato Ambuludí-Hualpa

La Ecuación 3 define la fuerza inicial de ajuste de los pernos sujetadores de la brida como porcentaje de la resistencia de prueba del perno (Hamrock, Jacobson, Schmid, 2000).

$$
\mathrm{F}_{i}=0,75 \times \sigma_{\mathrm{p}} \times A_{\text {sel_pern_suj_brid }} .
$$

Dónde:

$\sigma_{p}:$ Resistencia de prueba del material del perno utilizado (Pa) $A_{\text {sel_pern_suj_brid }}$ : Área seleccionada del perno sujetador de la brida $\left(\mathrm{m}^{2}\right)$

La Ecuación 4 calcula el espesor del soporte de la brida inferior, fundamentada en el esfuerzo flector de una viga corta en voladizo (Myzka, 2012).

$$
\mathrm{e}_{\text {sop_brid_inf }}=\sqrt{\frac{6 \times \mathrm{Mf}_{\text {brida }}{ }^{*} \mu s_{\text {brid }}}{\pi^{*} \mathrm{D}_{\text {int_cam }}{ }^{*} \sigma_{\text {y_mat_brid }}}} .
$$

Dónde:

$M f_{\text {brida }}$ :Momento flector de la base de la brida (N.m)

$\mu s_{\text {brid }}$ :Coeficiente de seguridad (adimensional)

$D_{\text {int_cam }}$ :Diámetro interior de la camisa $(\mathrm{m})$

$\sigma_{y_{-} \text {mat_brid }}$ Límite de fluencia del material de la brida (Pa)

La Ecuación 5 determina la presión resultante de la columna de descarga de la bomba (Mott, Untener, J. (2015); (Cengel, Cimbala, 2006).

$$
\operatorname{Pr}_{\mathrm{b}}=\delta \times \mathrm{g} \times \mathrm{H}
$$


Dónde:

$\delta:$ Densidad del agua $=1000 \mathrm{~kg} / \mathrm{m} 3$

g: Aceleración gravitacional $=9.81 \mathrm{~m} / \mathrm{s} 2$

$H$ : Altura de descarga $(\mathrm{m})$

La Ecuación 6 determina el número de pernos que sujetan la brida basado en el diámetro de la camisa (DNBrida, 2019).

$$
N_{\text {per_suj_brid }}=f\left(D_{\text {int_cam }}\right)
$$

En la Tabla 2 se cita el número de pernos recomendados de acuerdo a los rangos posibles de diámetro interno de la camisa.

La Ecuación 7 asigna el valor del límite de fluencia del material seleccionado para la brida. En la Tabla 3 se enlista las opciones de materiales propuestos (Hibbeler, 2011).

$$
\sigma_{\text {flu_mat_brid }}=f(\text { mat_brid) }
$$

\section{Obtención del algoritmo mediante MGD}

El método de grafos dicromáticos se aplica con el fin de obtener algoritmos para resolver problemas de cómputo. Existen varias publicaciones desde 1997 que mediante la teoría de grafos representan estructuras de modelos, situaciones, problemas y algoritmos, que se van transformando sucesivamente (Rodríguez, Marrero \& Lazo, 2012).

Una vez generado el MMl se procede a obtener su algoritmo de solución. En primera instancia se transcribe el MMI en el software de análisis matemático TK Solver®, luego se lo exporta al j2gml, software para la solución de problemas de cómputo basado en el MGD, desarrollado por (Escalona, Romero, Marrero \& Rodríguez, 2013), con la 
finalidad de obtener el grafo del algoritmo de solución.

En el Gráfico 5 se sintetizan las fases para la aplicación del MGD, proceso que parte desde la obtención del grafo del modelo matemático hasta el algoritmo de solución del problema de cómputo.

Como referencia metodológica, se expone el proceso de aplicación del Método de Grafos Dicromáticos al modelo matemático planteado para la brida inferior. Así, como primer paso se construye el grafo del modelo, el cual se exhibe en el Gráfico 6. En él, los vértices representan las variables y ecuaciones, diferenciándose únicamente por su color, amarillo y verde respectivamente. Las aristas representan las relaciones entre variables y ecuaciones.

Planteado el problema de cómputo, del grafo del modelo se eliminan las variables de entrada, pues son datos conocidos, obteniendo el grafo de la situación que se muestra en el Gráfico 7. Para la brida inferior se declaran las variables de entrada: diámetro interior de la camisa, densidad de fluido, aceleración gravitacional, cabezal de descarga, material de la brida, coeficiente de seguridad del perno, material del perno sujetador de la brida, grado del perno sujetador y caudal. A partir del grafo de la situación, se suprimen las componentes conexas que no contienen variables de salida que aporten a la solución, obteniendo el grafo del problema que se exhibe en el Gráfico 8.

A partir del grafo del problema se genera el grafo del problema pareado que se muestra en el Gráfico 9. Del grafo del problema se observa que algunas variables no tienen predefinida una solución, es decir, una arista que se dirija desde el vértice de una ecuación hacia el vértice de una variable. Entonces, se propone un pareo complementario, convirtiendo aristas en arcos de conexión exclusiva. Como consecuencia se obtiene un pareo máximo, compatible y realizable a partir de la existencia o no de deficiencias y variables libres. En un problema pareado, los arcos del pareo indican cuál relación podría computar a cuál incógnita. En este conjunto máximo de problemas elementales, no hay orden alguno establecido. El grafo del problema pareado generalmente es un grafo parcialmente orientado. 
En seguida, el grafo del resolvente, mostrado en el Gráfico 10, se obtiene del grafo del problema pareado, orientando todas las aristas remanentes; estas aristas se convierten en arcos que van de las incógnitas a las relaciones. En este caso es un problema plenamente pareado, es decir se encuentra en su forma canónica.

Finalmente, a partir del grafo resolvente se obtiene el grafo del algoritmo, exhibido en el Gráfico 11, mismo que muestra el orden a seguir para resolver el problema por vía algebraica y numérica. Para esto, se suprimen los problemas elementales no necesarios para obtener las salidas y se inicia cada ciclo con una meta elemental (Escalona, Romero, Marrero \& Rodríguez, 2013).

En la Tabla 4 se representa la estructura del algoritmo de solución de la brida inferior mediante el diagrama de Nassi-Schneiderman en bloque de procesos.La metodología expuesta para la brida inferior se replica para la bomba de pistón en conjunto, obteniendo su grafo de algoritmo de solución que se muestra en el Gráfico 12. Bajo la luz de esto, se valida la solución del problema de cómputo en base a las variables de entrada propuestas: caudal $(\mathrm{Q})$ y cabezal de descarga $(H)$.

\section{Programación del modelo en Microsoft Excel $\AA$ y enlace con Autodesk Inventor ${ }^{\circledR}$}

El MMI obtenido es programado en una hoja de cálculo de Microsoft Excel® mediante el módulo Visual Basic con el objeto de construir una interfaz de interacción en donde se introducen los datos de entrada, $\mathrm{Q}$ y $\mathrm{H}$, para realizar el cálculo de la geometría completa del prototipo.

Así, para cada componente de la bomba: camisa, brida inferior, brida superior, vástago, pistón y accesorios (acoples de succión y descarga, tuercas, pernos, válvulas, entre otros) se generan 2 hojas de cálculo. La primera detalla los datos geométricos y la segunda datos funcionales, ambos, producto de la ejecución del algoritmo matemático obtenido.

Se elaboran también tres hojas de cálculo generales, donde la primera es netamente informativa y explica las consideraciones a tomar en cuenta para la ejecución del aplicativo, relacionados con los datos de entrada, datos de salida y restricciones. La segunda se constituye como la interfaz, Gráfico 13, donde se introducen los parámetros 
Byron Solorzano-Castillo; Jairo Castillo-Calderón; Renato Ambuludí-Hualpa

de entrada y mediante botones programados se ejecuta la totalidad del cálculo de los parámetros de diseño de la bomba y la vinculación con el Autodesk Inventor® para generar el modelo virtual 3D; otros botones permiten interactuar con las hojas de cálculo explicadas anteriormente. Finalmente, la tercera exhibe un informe final resumido del prototipo de bomba, donde constan datos de diseño, de construcción y listado de materiales de cada componente.

A continuación, se vincula las variables paramétricas, generadas de forma automática en el software CAD, con la hoja de cálculo, donde se ejecuta la aplicación para distintos valores de caudal y cabezal de descarga obteniendo el correspondiente modelo virtual de la bomba. El intervalo de caudal está comprendido entre 0 y $1,25 \mathrm{l} / \mathrm{s}$, y el del cabezal de descarga, entre 0 a 100 mca; estas consideraciones se fundamentan en la velocidad lineal recomendada en bombas de pistón y en la resistencia máxima de los materiales que conforman la camisa.

\section{RESULTADOS Y DISCUSIÓN}

En el presente apartado se exponen los resultados del uso de la aplicación para dos casos de estudio en condiciones de diseño totalmente opuestas; el primero considera requerimientos de alto caudal y bajo cabezal de descarga, y el segundo, bajo caudal y alto cabezal de descarga.

En primera instancia se ingresa un caudal de 1,25 L/s y un cabezal de descarga de 10 mca, obteniéndose una camisa de PVC, bridas de $6 \mathrm{~mm}$ de espesor y 12 pernos de sujeción, el vástago con un diámetro de $18 \mathrm{~mm}$ y los accesorios de entrada y salida de agua con un diámetro interno de $66,16 \mathrm{~mm}$; el Gráfico 14 exhibe los resultados constructivos de la bomba.

Las dimensiones e información constructiva para la totalidad de componentes de la bomba, tales como: camisa, brida superior, brida inferior, vástago, pistón, accesorio de succión, accesorio de descarga y pernos, se detallan por hojas de cálculo independientes, incluyendo además para cada uno de ellos planos donde constan las variables parametrizadas. Así, para ejemplificar los resultados, el Gráfico 15 muestra 
los datos geométricos que conforman la brida inferior otorgada por la aplicación.

El prototipo virtual obtenido del primer caso de estudio se detalla en el Gráfico 16. A primera vista, se observa el número de pernos de las tapas laterales de la camisa, o bridas, los cuales son función del diámetro de la camisa y del pistón; en este caso son pernos con un diámetro de $4 \mathrm{~mm}$. Los accesorios de entrada y salida del fluido son de diámetro relativamente considerable debido al caudal de diseño.

En el segundo caso de estudio se ingresa un caudal de $0,25 \mathrm{~L} / \mathrm{s}$ y un cabezal de descarga de $100 \mathrm{mca}$, obteniéndose acero inoxidable 304 como material para la camisa, bridas de acero SAE 1015 con un espesor de 6 mm y 8 pernos de sujeción, el vástago de acero inoxidable con un diámetro de $31,75 \mathrm{~mm}$ y los accesorios de entrada y salida de agua de hierro galvanizado con un diámetro interno de 36,7 mm.; el Gráfico 17 detalla los resultados constructivos de la bomba.

El prototipo virtual de la bomba obtenido se muestra en el Gráfico 18. A diferencia del caso anterior, se observa de manera notoria un menor número de pernos en las bridas. Ahora, los accesorios de entrada y salida del fluido con un diámetro menor debido a la disminución del caudal de diseño.

La aplicación fue concebida con base en metodologías antes validadas, como la utilizada en (Solórzano, 2014), donde se construye un MMI para un compresor o la presentada en (Marrero, 2009). donde se plantea el diseño paramétrico basado en modelos matemáticos de máquinas para la construcción sostenible de viviendas.

El MMI de la bomba contiene 90 relaciones y 140 variables, donde se integran submodelos geométricos, de resistencia de materiales, hidráulicos, además del uso de 22 parámetros de diseño. El algoritmo de solución del modelo planteado se lo obtuvo utilizando el MGD debido a su sencillez, su grado de terminación y la certidumbre de sus resultados. Además, en (Marrero \& Martínez, 2008), se afirma que el método permite aprovechar las particularices teóricas de los grafos dicromáticos durante el proceso de solución de problemas, pasando por un proceso rigurosamente ordenado a partir de la caracterización del problema y su correcta formulación hasta la obtención del algoritmo. 
El algoritmo de solución tiene como variables de entrada el caudal y la altura piezométrica, que son los parámetros más comunes al momento de seleccionar una bomba, mismos que están comprendidos entre 0 y $1.25 \mathrm{l} / \mathrm{s}$, así como de 0 a 100 mca., respectivamente; esto debido a que representan rangos de aplicabilidad comunes para este tipo de bombas. Otro aspecto importe es el material de la camisa, como son el PVC y el acero inoxidable, puesto que son comunes en el medio.

La programación del algoritmo se ha desarrollado en Microsoft Excel $2013 \AA$, debido a la experiencia en el manejo y la facilidad de vinculación con Autodesk Inventor 2017®, que fue el programa elegido para obtener el prototipo virtual de la bomba. Queda abierta la posibilidad de programarlo directamente en el software de diseño 3D, utilizando la función llogic18 que viene incorporada, pero resulta más compleja.

La aplicación junto con la metodología empleada, permiten la obtención de un primer modelo virtual, el cuál puede ser analizado y/o modificado de acuerdo a criterios del diseñador; para el caso en concreto, representa una herramienta para la síntesis del diseño de la bomba de pistón. Además, la metodología planteada se espera, sirva como guía para el desarrollo de futuras aplicaciones destinas al diseño paramétrico de las múltiples máquinas y/o piezas que existen el campo de la ingeniería mecánica.

Es perentorio mencionar que la presente aplicación representa una versión inicial, la cual podría vincularse a otras herramientas CAD, la incursión del análisis de elementos finitos, simuladores dinámicos, optimizadores, entre otros, con el fin de profundizar el estudio y obtener diseños finales con mayor nivel de complejidad y detalle. Asimismo, podría incorporarse a la aplicación, como trabajos futuros, aspectos relevantes como el tiempo de manufactura, prototipos virtuales físicos a través de impresión 3D y análisis de coste.

\section{CONCLUSIONES}

La metodología propuesta, así como el uso del programa Excel y el software Autodesk Inventor $\AA$, permiten obtener modelos virtuales tridimensionales flexibles, dinamizando el proceso de diseño de máquinas y piezas; las nuevas geometrías se generan de 
manera automática acordes al modelo matemático empleado y a los diferentes parámetros de operación.

Para el caso concreto, la aplicación desarrollada trabaja un modelo matemático con un número considerable de relaciones y variables, 90 y 140 respectivamente, las cuales interactúan de forma armónica con la simple introducción de los dos parámetros de diseño de entrada: caudal y altura piezométrica; los prototipos virtuales de la bomba de pistón varían según estos criterios, lo que da certeza de su correcta funcionalidad.

El Método de Grafos Dicromáticos constituye una herramienta eficaz para caracterizar y resolver de modo confiable problemas de cómputo que se planteen en diseño paramétrico, sin importar que el modelo matemático sea de grandes dimensiones, siempre que éste gobierne aceptablemente el comportamiento del objeto de diseño.

\section{FINANCIAMIENTO}

No monetarios

\section{AGRADECIMIENTO}

Los autores agradecen a las autoridades locales de los sectores donde se ejecutó la investigación, y a la Universidad Nacional de Loja, por el apoyo técnico, científico realizado a la presente investigación. 
Revista Arbitrada Interdisciplinaria KOINONIA

Año V. Vol V. №9. Enero - Junio 2020

Hecho el depósito de Ley: FA2016000010

ISSN: 2542-3088

FUNDACIÓN KOINONIA (F.K). Santa Ana de Coro. Venezuela.

Byron Solorzano-Castillo; Jairo Castillo-Calderón; Renato Ambuludí-Hualpa

\section{ANEXOS}

Tabla 1

Especificaciones técnicas de la bomba

\begin{tabular}{ll}
\hline Característica & Descripción \\
\hline Potencia de accionamiento & $0,57 \mathrm{hp}$ \\
Frecuencia de rotación & $14 \mathrm{rpm}$ \\
Caudal & $0,54 \mathrm{l} / \mathrm{s}$ \\
Altura de descarga & $12 \mathrm{~m}$ \\
Diámetro y carrera del pistón & $0,15 \mathrm{~m} \mathrm{x} \mathrm{0,14} \mathrm{m}$ \\
Tipo de válvulas & Esfera \\
Material del pistón & Suela \\
\hline
\end{tabular}

Fuente: Pineda (2014).

Gráfico 1. Esquema de la bomba de pistón y sus partes
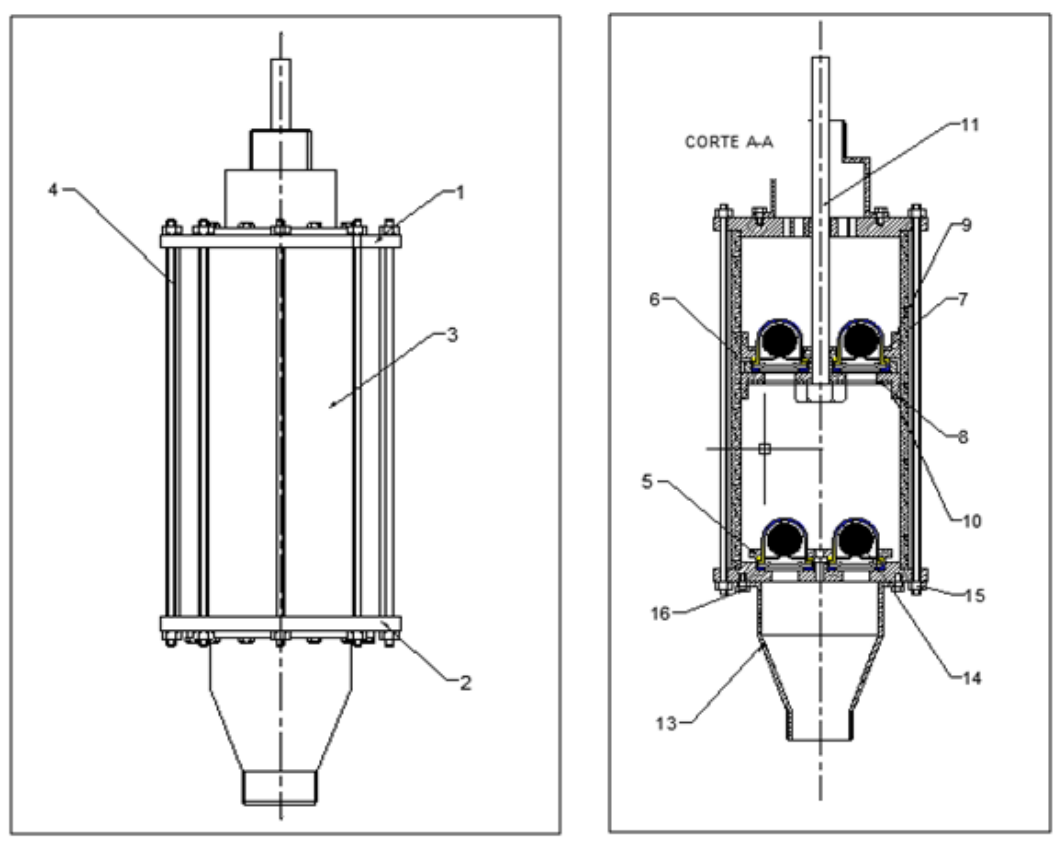

Fuente: Pineda (2014). 
Revista Arbitrada Interdisciplinaria KOINONIA

Año V. Vol V. №9. Enero - Junio 2020

Hecho el depósito de Ley: FA2016000010

ISSN: 2542-3088

FUNDACIÓN KOINONIA (F.K). Santa Ana de Coro. Venezuela.

Byron Solorzano-Castillo; Jairo Castillo-Calderón; Renato Ambuludí-Hualpa

Gráfico 2. Plano de la brida inferior en dos dimensiones.

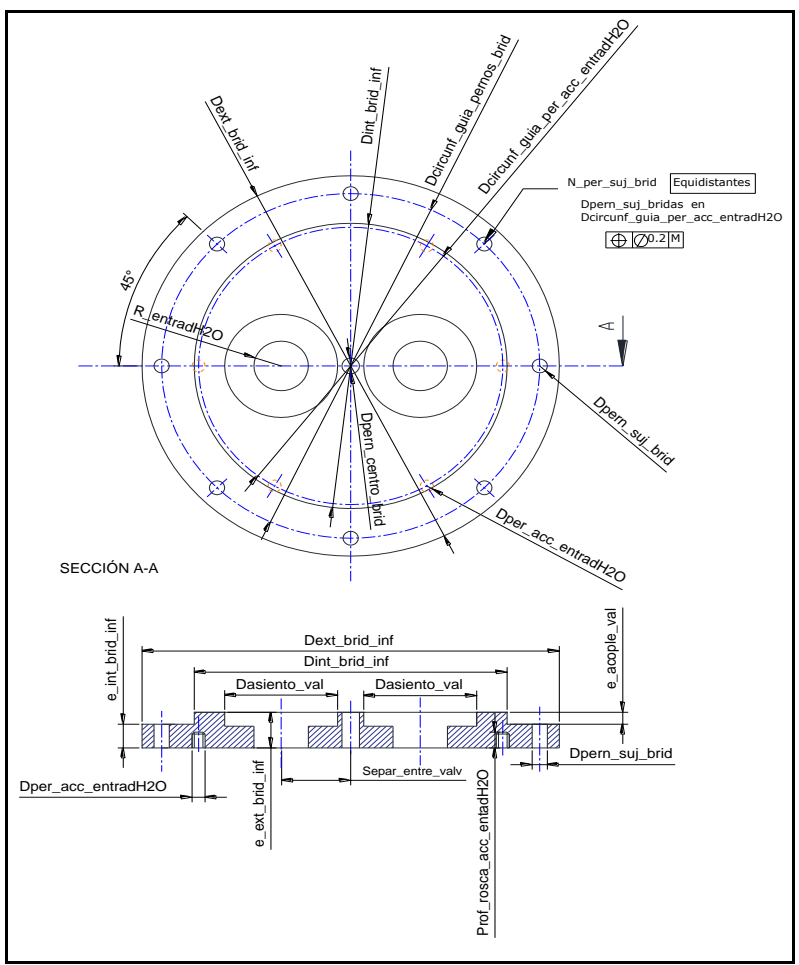

Fuente: Autores, 2019

Gráfico 3. Brida inferior generada en 3D.

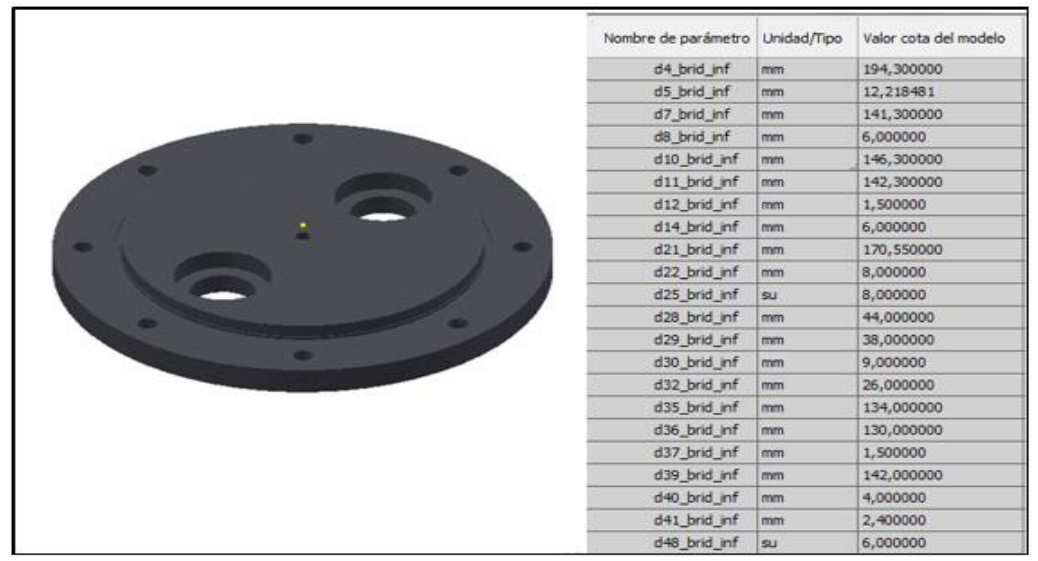

Fuente: Autores, 2019 
Revista Arbitrada Interdisciplinaria KOINONIA

Año V. Vol V. №9. Enero - Junio 2020

Hecho el depósito de Ley: FA2016000010

ISSN: 2542-3088

FUNDACIÓN KOINONIA (F.K). Santa Ana de Coro. Venezuela.

Byron Solorzano-Castillo; Jairo Castillo-Calderón; Renato Ambuludí-Hualpa

Gráfico 4. (a) Vista en 3D de los componentes internos de la bomba, (b) Vista en 3D de la bomba con corte de sección.

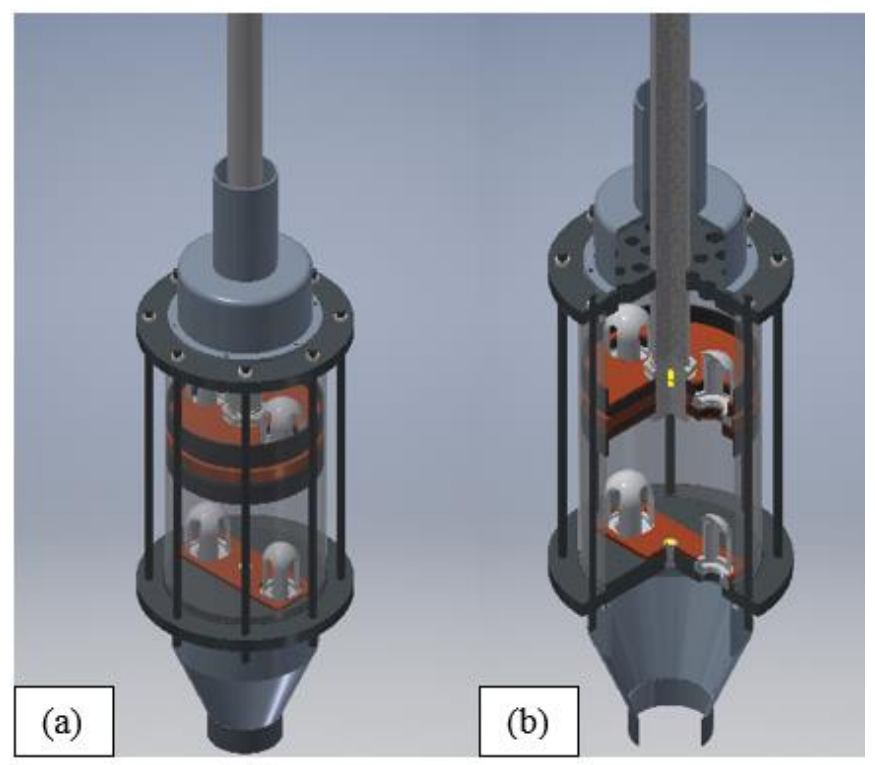

Fuente: Autores, 2019

Tabla 2. Número de pernos sujetadores de la brida en función del diámetro de la camisa

\begin{tabular}{cc}
\hline Diámetro interno de la camisa & Número de pernos sujetadores de la brida \\
\hline$>12 ?=76,2$ & 4 \\
$>76,2 ?=203,2$ & 8 \\
$>203,2 ?=355,6$ & 12 \\
\hline
\end{tabular}

Fuente: DNBrida (2019).

Tabla 3. Límite a la fluencia en función de los materiales de la brida

\begin{tabular}{cc}
\hline Material de la brida & Límite de fluencia \\
\hline Acero SAE 1010 & 280 \\
Acero SAE 1015 & 190 \\
Acero SAE 1020 & 210 \\
\hline
\end{tabular}

Fuente: Hibbeler, (2011). 
Revista Arbitrada Interdisciplinaria KOINONIA

Año V. Vol V. N9. Enero - Junio 2020

Hecho el depósito de Ley: FA2016000010

ISSN: 2542-3088

FUNDACIÓN KOINONIA (F.K). Santa Ana de Coro. Venezuela.

Byron Solorzano-Castillo; Jairo Castillo-Calderón; Renato Ambuludí-Hualpa

Gráfico 5. Esquema del MGD

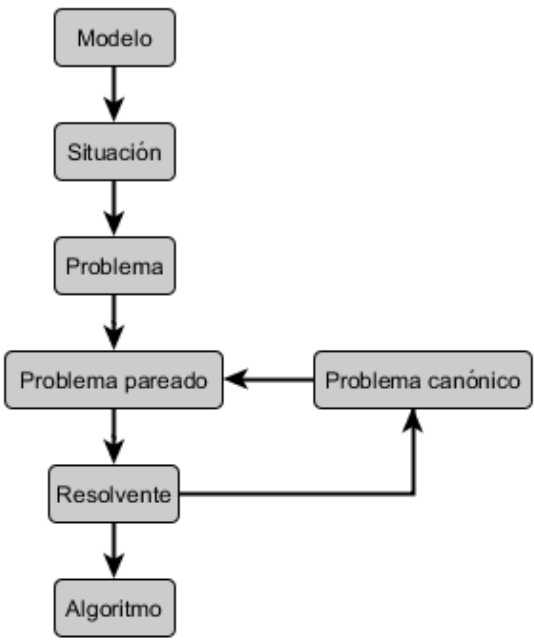

Fuente: Escalona, Romero, Marrero \& Rodríguez, 2013.

Gráfico 6. Grafo del modelo matemático de la brida inferior.

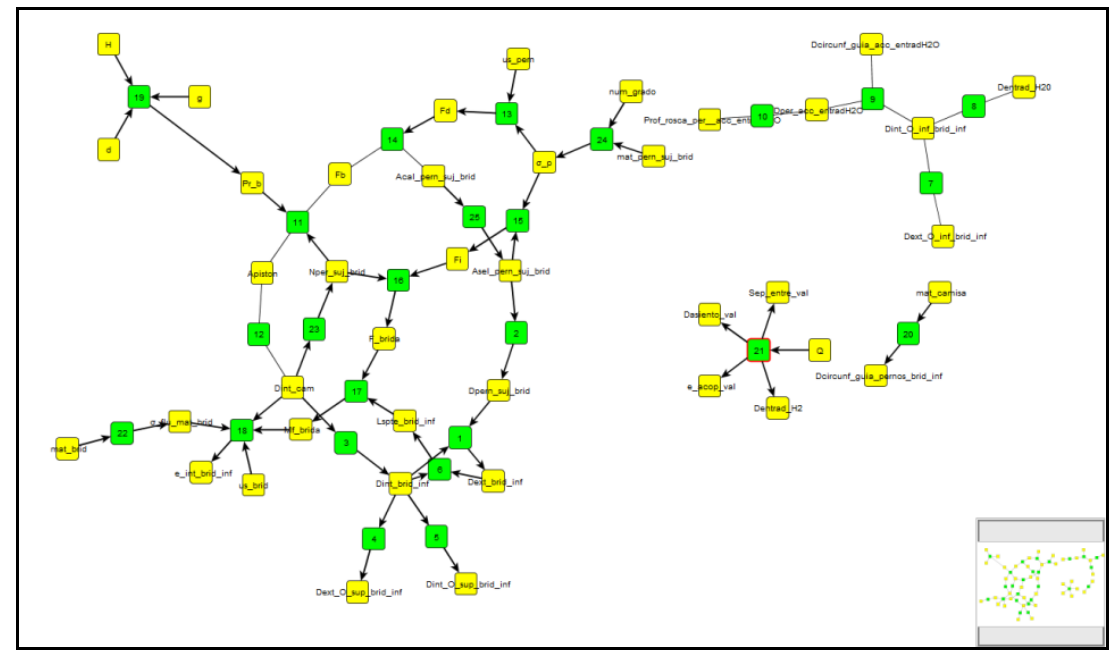

Fuente: Autores, 2019 
Revista Arbitrada Interdisciplinaria KOINONIA

Año V. Vol V. №9. Enero - Junio 2020

Hecho el depósito de Ley: FA2016000010

ISSN: 2542-3088

FUNDACIÓN KOINONIA (F.K). Santa Ana de Coro. Venezuela.

Byron Solorzano-Castillo; Jairo Castillo-Calderón; Renato Ambuludí-Hualpa

Gráfico 7. Grafo de la situación de la brida inferior.

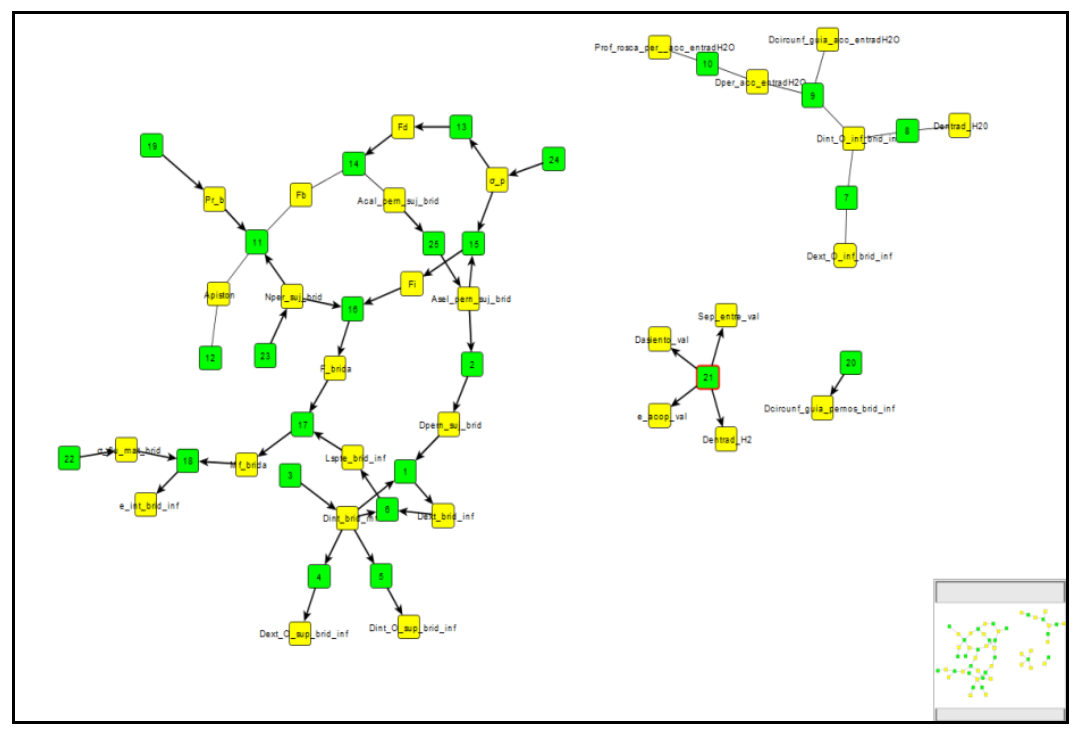

Fuente: Autores, 2019

Gráfico 8. Grafo del problema de la brida inferior.

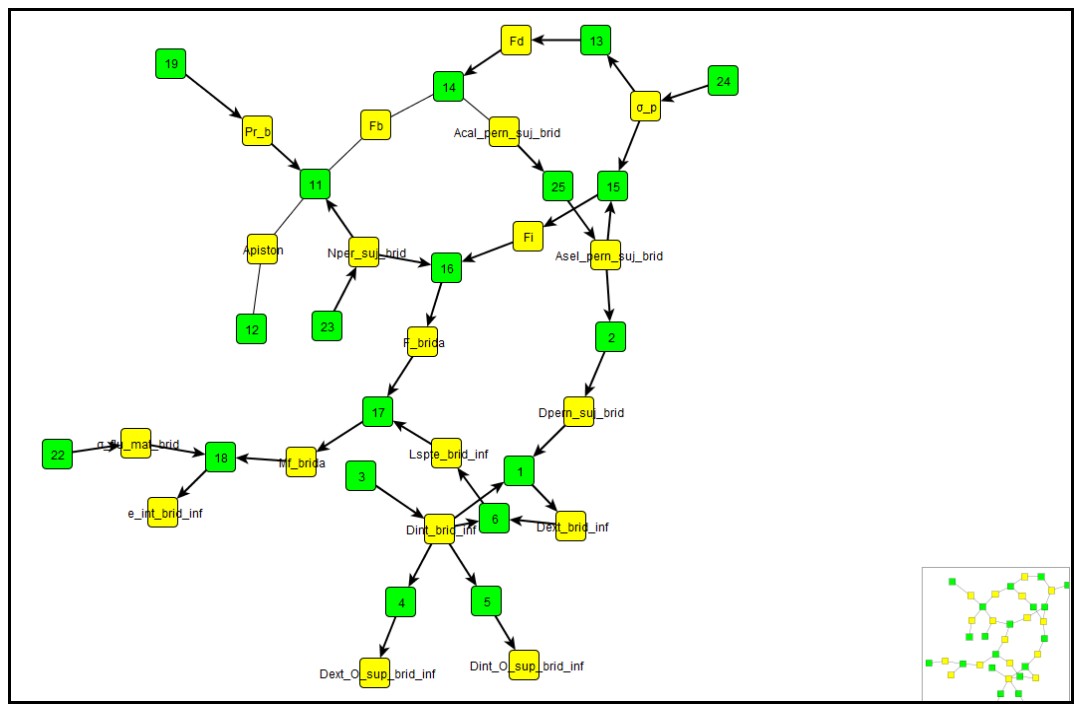

Fuente: Autores, 2019 
Revista Arbitrada Interdisciplinaria KOINONIA

Año V. Vol V. N9. Enero - Junio 2020

Hecho el depósito de Ley: FA2016000010

ISSN: 2542-3088

FUNDACIÓN KOINONIA (F.K). Santa Ana de Coro. Venezuela.

Byron Solorzano-Castillo; Jairo Castillo-Calderón; Renato Ambuludí-Hualpa

Gráfico 9. Grafo del problema pareado de la brida inferior.

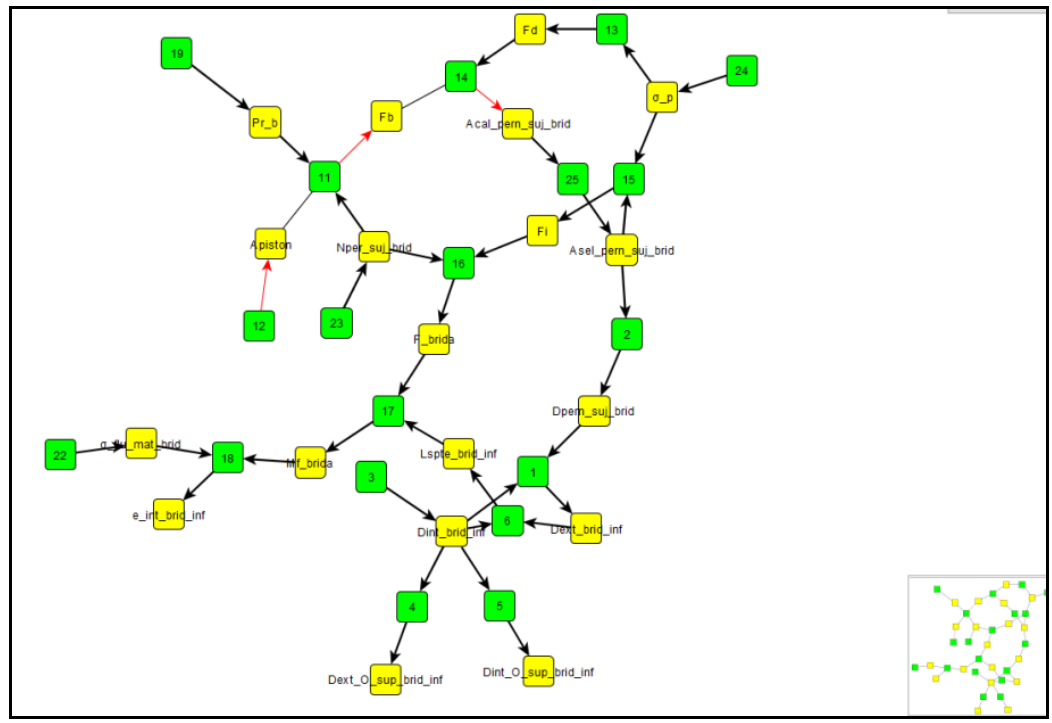

Fuente: Autores, 2019 
Revista Arbitrada Interdisciplinaria KOINONIA

Año V. Vol V. №9. Enero - Junio 2020

Hecho el depósito de Ley: FA2016000010

ISSN: 2542-3088

FUNDACIÓN KOINONIA (F.K). Santa Ana de Coro. Venezuela.

Byron Solorzano-Castillo; Jairo Castillo-Calderón; Renato Ambuludí-Hualpa

Gráfico 10. Grafo del resolvente de la brida inferior.

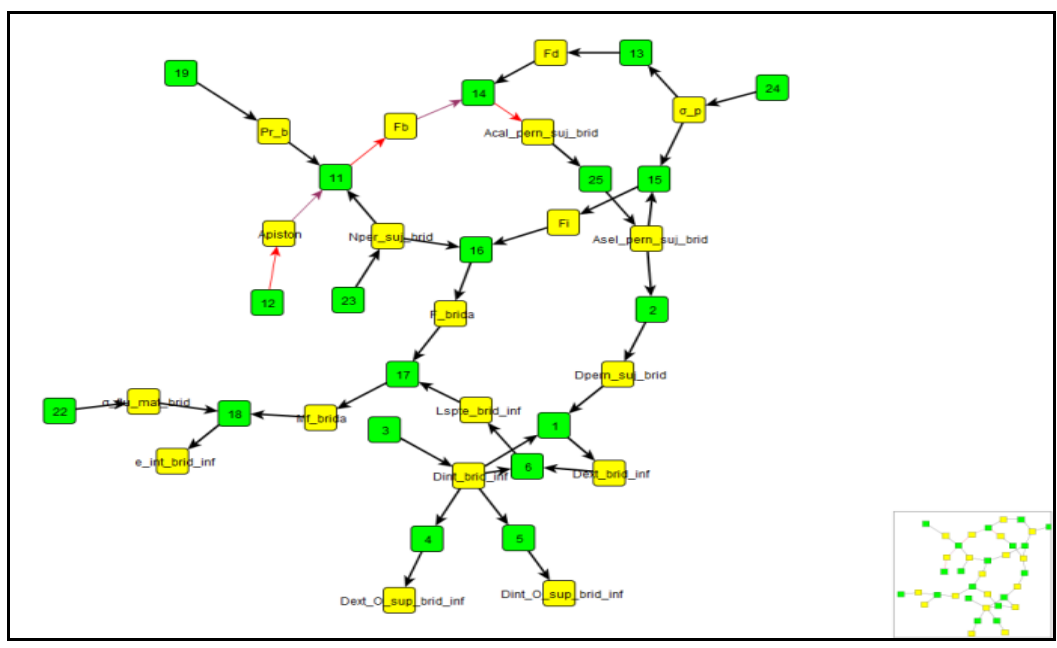

Fuente: Autores, 2019

Gráfico 11. Grafo del algoritmo de solución de la brida inferior.

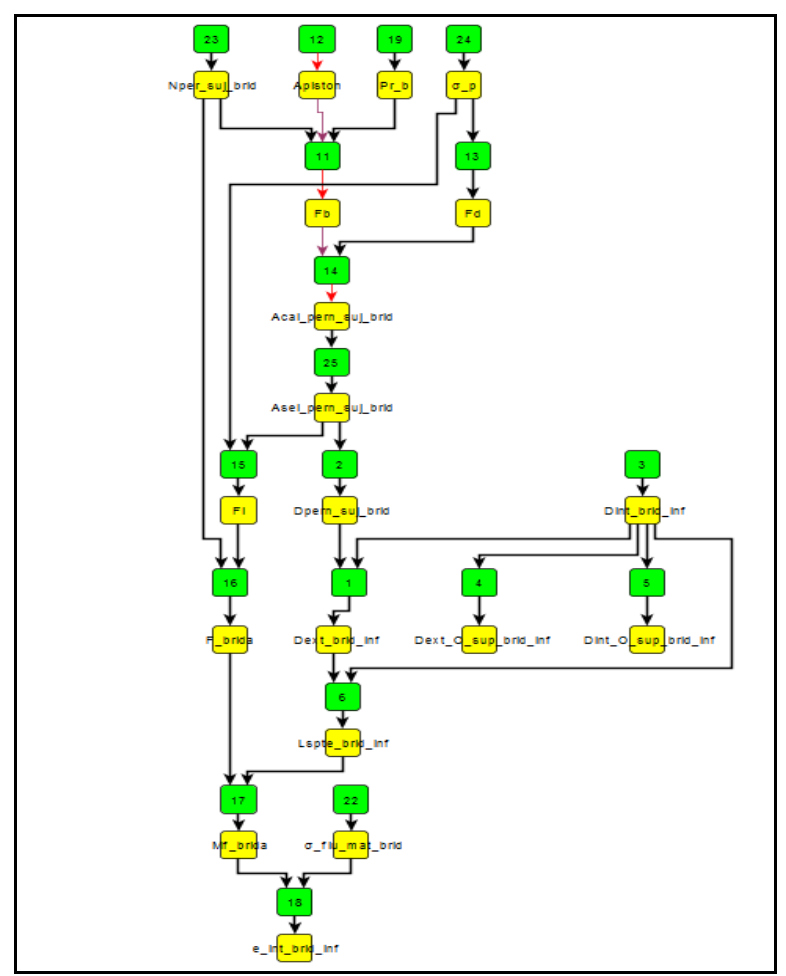

Fuente: Autores, 2019 
Revista Arbitrada Interdisciplinaria KOINONIA

Año V. Vol V. №9. Enero - Junio 2020

Hecho el depósito de Ley: FA2016000010

ISSN: 2542-3088

FUNDACIÓN KOINONIA (F.K). Santa Ana de Coro. Venezuela.

\section{Byron Solorzano-Castillo; Jairo Castillo-Calderón; Renato Ambuludí-Hualpa}

Tabla 4. Estructograma de Nassi-Scheirman para el algoritmo de solución de la brida inferior.

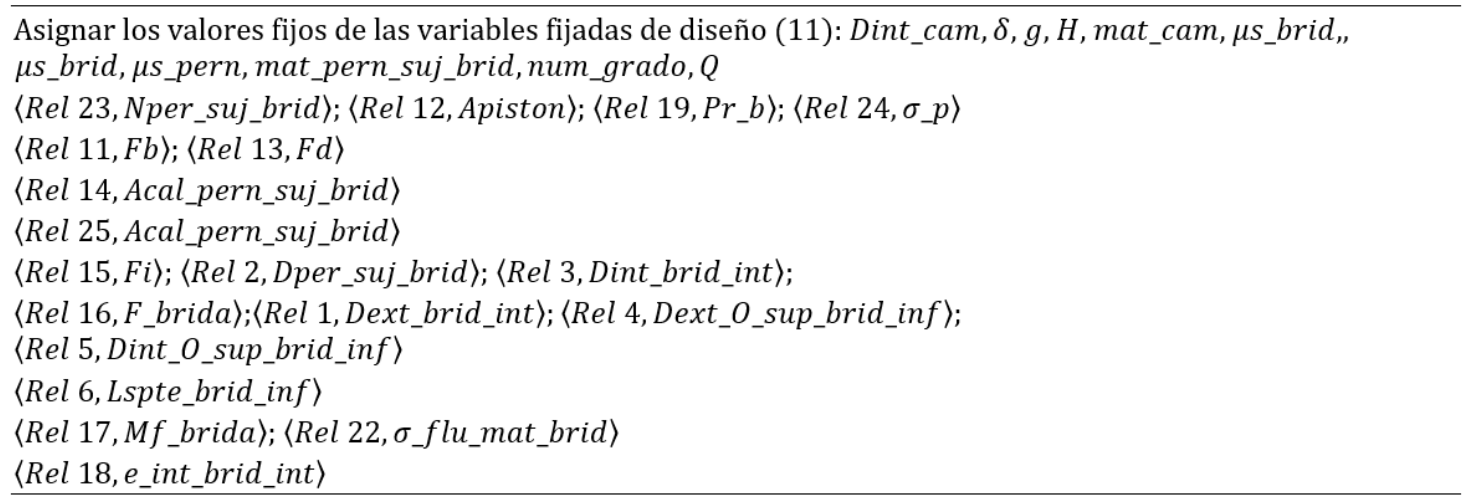

Fuente: Autores, 2019

Gráfico 12. Grafo del algoritmo de solución de la bomba de pistón.

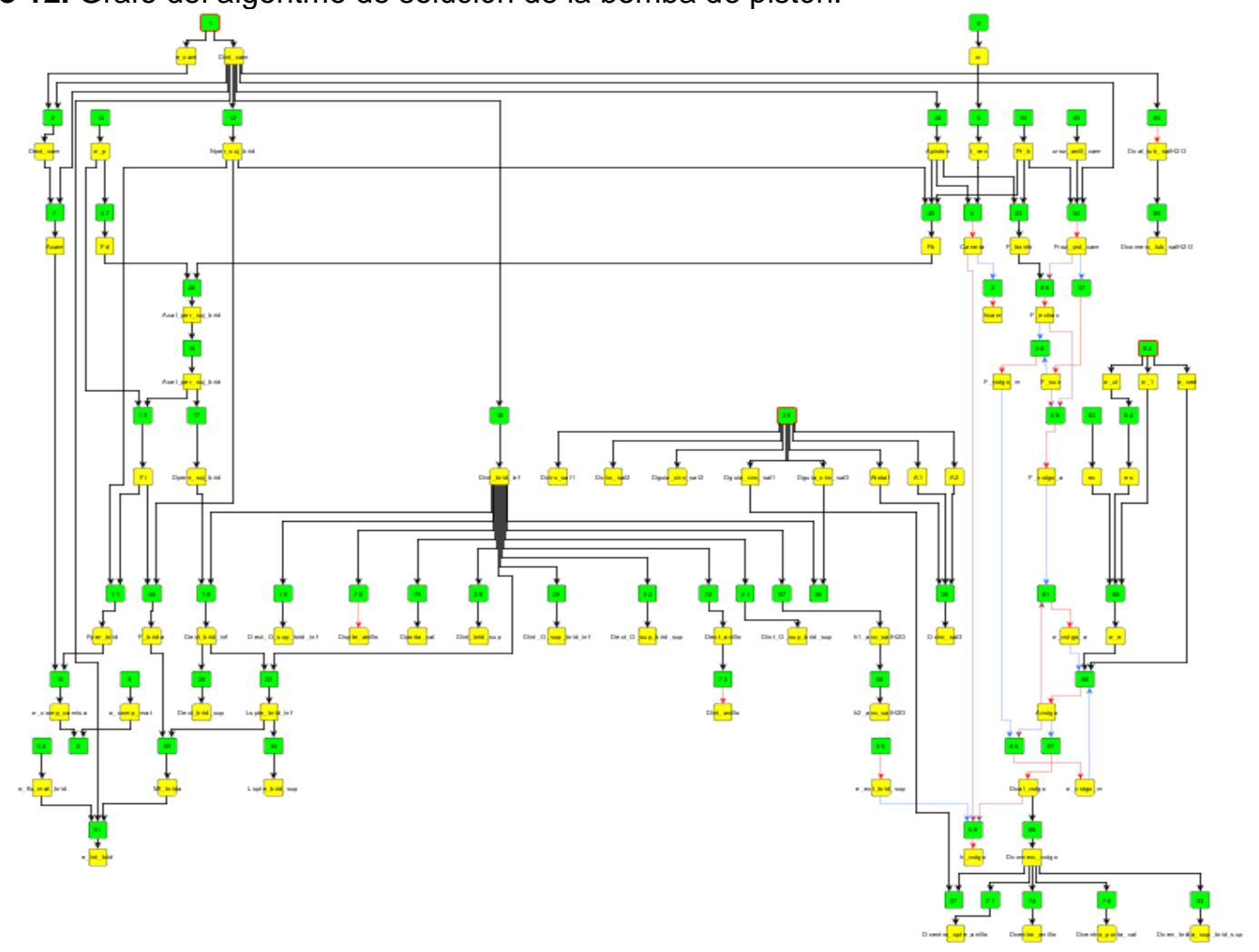

Fuente: Autores, 2019 
Revista Arbitrada Interdisciplinaria KOINONIA

Año V. Vol V. №9. Enero - Junio 2020

Hecho el depósito de Ley: FA2016000010

ISSN: 2542-3088

FUNDACIÓN KOINONIA (F.K). Santa Ana de Coro. Venezuela.

Byron Solorzano-Castillo; Jairo Castillo-Calderón; Renato Ambuludí-Hualpa

Gráfico 13. Interfaz para ingreso de datos de diseño.

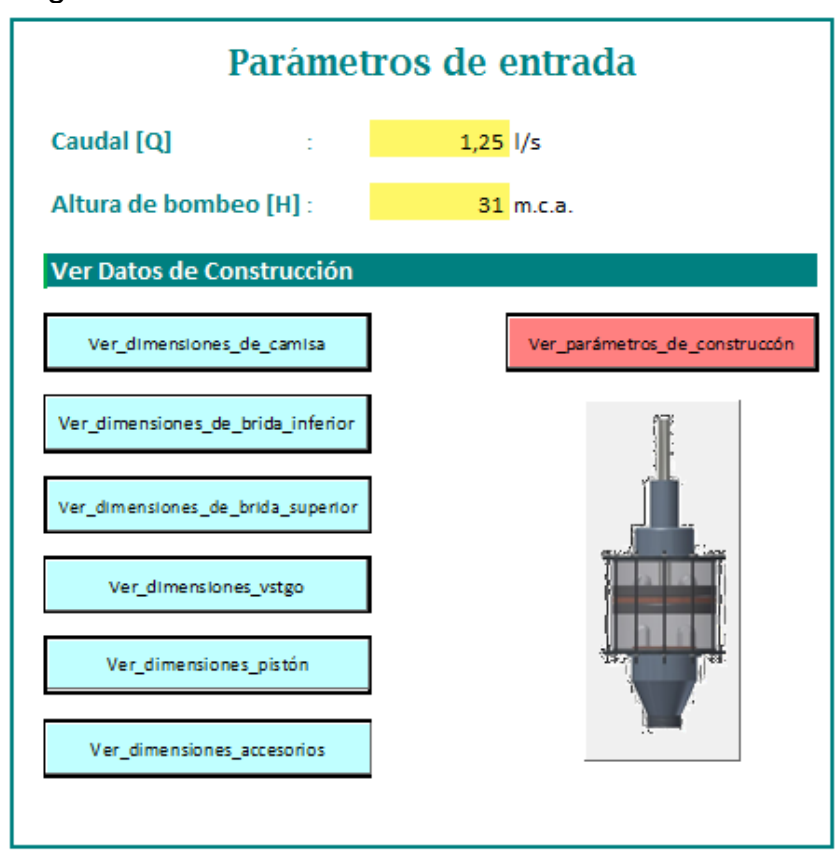

Fuente: Autores, 2019

Gráfico 14. Parámetros generales de construcción de los componentes de la bomba para el primer caso de estudio.

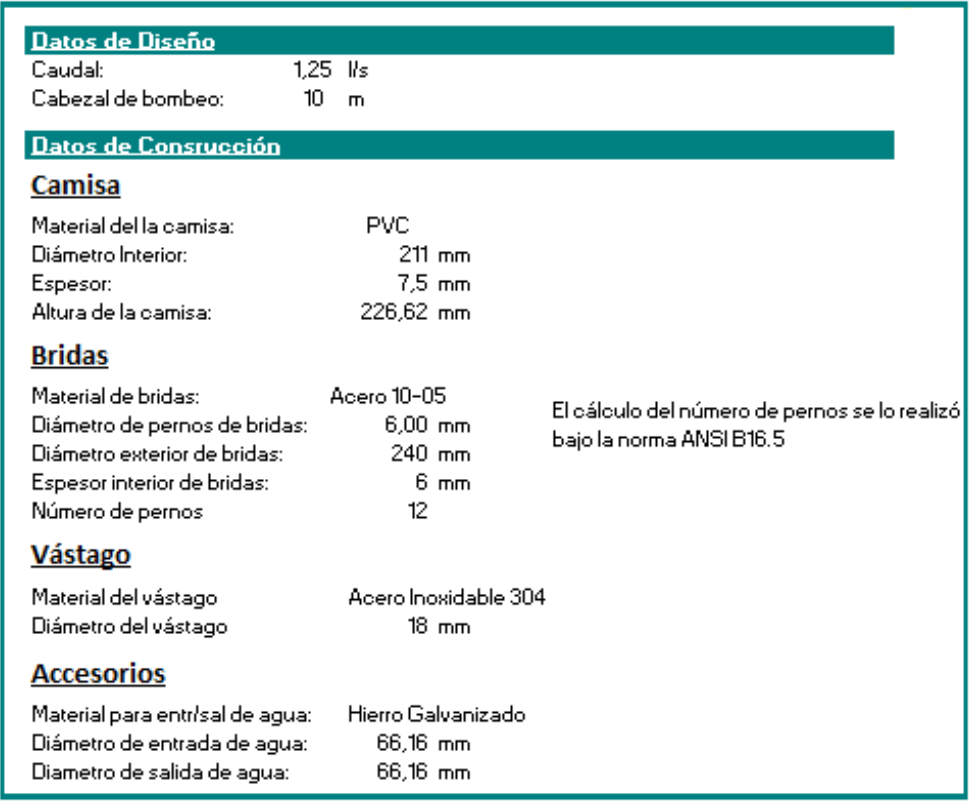

Fuente: Autores, 2019 
Revista Arbitrada Interdisciplinaria KOINONIA

Año V. Vol V. №9. Enero - Junio 2020

Hecho el depósito de Ley: FA2016000010

ISSN: 2542-3088

FUNDACIÓN KOINONIA (F.K). Santa Ana de Coro. Venezuela.

Byron Solorzano-Castillo; Jairo Castillo-Calderón; Renato Ambuludí-Hualpa

Gráfico 15. Resultados de geometría de la brida inferior obtenidos desde la aplicación; $H=10$ mea; $Q=1,25 \mathrm{l} / \mathrm{s}$

\section{Datos Geométricos-Brida Inferior}

\begin{tabular}{|c|c|c|c|}
\hline Simblogía & Medida & Unidades & Comentario \\
\hline Dext_brid_inf & 240 & $\mathrm{~mm}$ & Diámetro exterior de la brida inferior \\
\hline e_int_brid_inf & 4,53 & $\mathrm{~mm}$ & Espesor interior de la brida inferior \\
\hline Dint_brid_inf & 211,00 & $\mathrm{~mm}$ & Diámetro interior de la brida inferior \\
\hline e_ext_brid_inf & 6 & $\mathrm{~mm}$ & Espesor exterior de la brida inferior \\
\hline Dext_O_sup_brid_inf & 216,00 & $\mathrm{~mm}$ & Diámetro exterior del Orings interior de brida inferior \\
\hline Dint_O_sup_brid_inf & 212 & $\mathrm{~mm}$ & Diámetro interior del Orings interior de brida inferior \\
\hline hran_orings_int_brid_inf & 1,5 & $\mathrm{~mm}$ & Altura de la ranura del Orings interior de brida inferior \\
\hline Dcentro_brid_inf & 6,00 & $\mathrm{~mm}$ & Diámetro central de la brida inferior \\
\hline Dcircunf_guia_pernos_brid_inf & 222,25 & $\mathrm{~mm}$ & Diam de la circun guia de los pernos de sujecion de brida \\
\hline Dperno_suj_bridas & 4 & $\mathrm{~mm}$ & Diámetro de pernos de sujecion de brida \\
\hline N_per_suj_brid & 12 & $\mathrm{ul}$ & Número de pernos de sujecion de brida \\
\hline Separ_entre_valv & 44,00 & $\mathrm{~mm}$ & Separación entre el centro de la válvula v el centro de la brida \\
\hline Dasiento_valv & 38,00 & $\mathrm{~mm}$ & Diámetro de asiento de la válvula \\
\hline e_acople_valv & 9,00 & & espesor de acople \\
\hline Dentrad_H2O & 26,00 & $\mathrm{~mm}$ & Diámetro de entrada de agua \\
\hline Dext_O_inf_brid_inf & 134,00 & $\mathrm{~mm}$ & Diámetro exterior del Orings exterior de brida inferior \\
\hline Dint_O_inf_brid_inf & 130 & $\mathrm{~mm}$ & Diámetro interior del Orings exterior de brida inferior \\
\hline hran_orings_inf_brid_inf & 1,5 & $\mathrm{~mm}$ & Altura de la ranura del Orings exterior \\
\hline D_circunf_guia_per_accesorH2O & 142,00 & $\mathrm{~mm}$ & Diam de la circun guia de los pernos del acc de entrada de agua \\
\hline Dper_acc_entradH2O & 4 & $\mathrm{~mm}$ & Diámetro de pernos de sujecion del acc de salida de agua \\
\hline Prof_rosca_per_acc_entradH2O & 2,40 & $\mathrm{~mm}$ & Profundiffad de rosca del pernos de sujecion del acc de salida de agua \\
\hline Num_per_acc_entradH2O & 6 & $\mathrm{ul}$ & Número de pernos de sujecion del acc de salida de agua \\
\hline
\end{tabular}

Fuente: Autores, 2019

Gráfico 16. Prototipo virtual 3D de la bomba de pistón, $H=10$ mea; $Q=1,25 \mathrm{l} / \mathrm{s}$, obtenido desde el aplicativo: a) Vista frontal, b) Vistas isométricas.

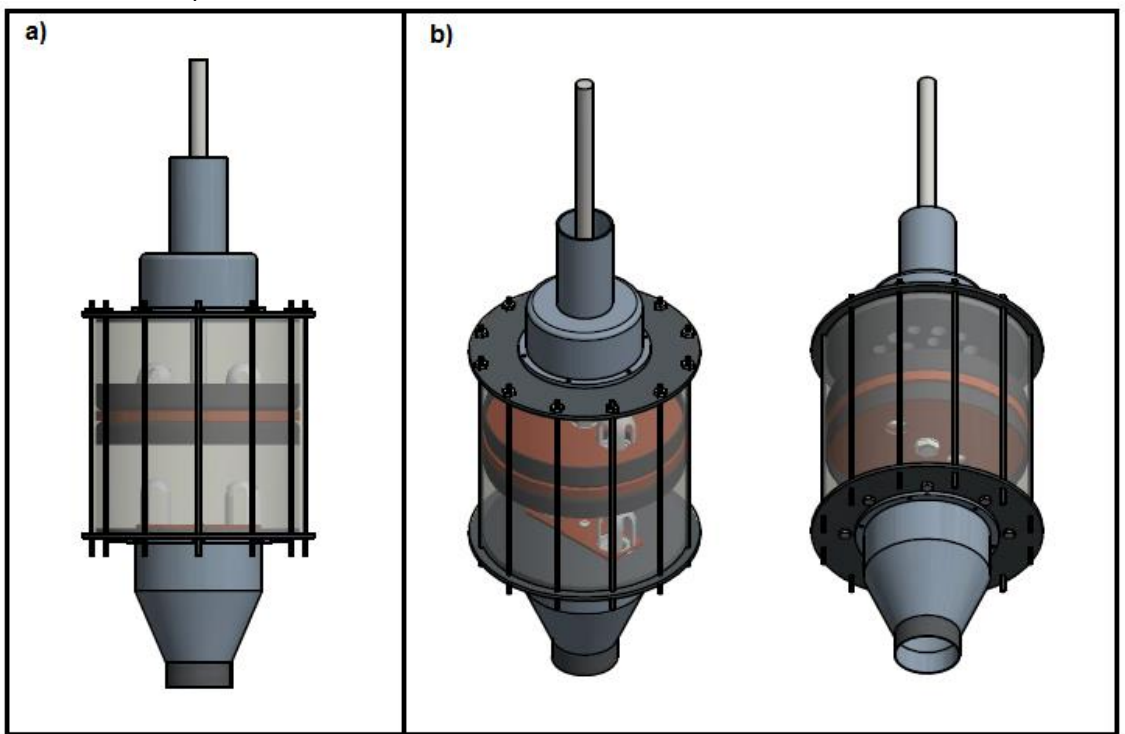

Fuente: Autores, 2019 
Revista Arbitrada Interdisciplinaria KOINONIA

Año V. Vol V. №9. Enero - Junio 2020

Hecho el depósito de Ley: FA2016000010

ISSN: 2542-3088

FUNDACIÓN KOINONIA (F.K). Santa Ana de Coro. Venezuela.

Byron Solorzano-Castillo; Jairo Castillo-Calderón; Renato Ambuludí-Hualpa

Gráfico 17. Parámetros generales de construcción de los componentes de la bomba para el segundo caso de estudio.

\begin{tabular}{|c|c|c|}
\hline \multicolumn{3}{|l|}{ Datos de Disefín } \\
\hline $\begin{array}{ll}\text { Caudal: } & 0.25 \\
\text { Cabezal de bombeo: } & 100\end{array}$ & \multicolumn{2}{|c|}{$\begin{array}{l}0.25 \mathrm{lts} \\
100 \mathrm{~m}\end{array}$} \\
\hline \multicolumn{3}{|l|}{ Datos de Constucsit } \\
\hline \multicolumn{3}{|l|}{ Camisa } \\
\hline \multicolumn{3}{|l|}{ Material del la camisa: } \\
\hline Diámetro Interior: & \multicolumn{2}{|c|}{$\begin{array}{r}\text { Acero Inoxidable } \\
141,3 \mathrm{~mm}\end{array}$} \\
\hline Espesor: & \\
\hline Altura de la camisa: & \multicolumn{2}{|l|}{$\begin{array}{r}3,4 \mathrm{~mm} \\
196,91 \mathrm{~mm}\end{array}$} \\
\hline \multicolumn{3}{|l|}{ Bridas } \\
\hline Material de bridas: & \multicolumn{2}{|l|}{ Acero 10-05 } \\
\hline Diámetro de pernos de bridas: & $6,00 \mathrm{~mm}$ & \multirow{3}{*}{$\begin{array}{l}\text { El cálculo del número de pernos se lo } \\
\text { realizó bajo la norma ANSI B16.5 }\end{array}$} \\
\hline Diámetro esterior de bridas: & $194,3 \mathrm{~mm}$ & \\
\hline Espesor interior de bridas: & $6 \mathrm{~mm}$ & \\
\hline Número de pernos & 8 & \\
\hline \multicolumn{3}{|l|}{ Vástago } \\
\hline Material del vástago & \multirow{2}{*}{\multicolumn{2}{|c|}{$\begin{array}{l}\text { Acero Inoxidable } 304 \\
31,750 \mathrm{~mm}\end{array}$}} \\
\hline Diámetro del vástago & & \\
\hline \multicolumn{3}{|l|}{ Accesorios } \\
\hline \multirow{2}{*}{$\begin{array}{l}\text { Material para entrisal de agua: } \\
\text { Diámetro de entrada de agua: }\end{array}$} & \multicolumn{2}{|c|}{ : Hierro Galvanizado } \\
\hline & \multicolumn{2}{|c|}{$\begin{array}{l}\text { Hierro Galvanizado } \\
36,7 \mathrm{~mm}\end{array}$} \\
\hline Diametro de salida de agua: & \multicolumn{2}{|c|}{$\begin{array}{l}36,7 \mathrm{~mm} \\
42,6 \mathrm{~mm}\end{array}$} \\
\hline
\end{tabular}

Fuente: Autores, 2019

Gráfico 18. Prototipo virtual $3 \mathrm{D}$ de la bomba de pistón, $H=100 \mathrm{mca} ; Q=0,25 \mathrm{l} / \mathrm{s}$, obtenido desde el aplicativo: a) Vista frontal, b) Vistas isométricas.

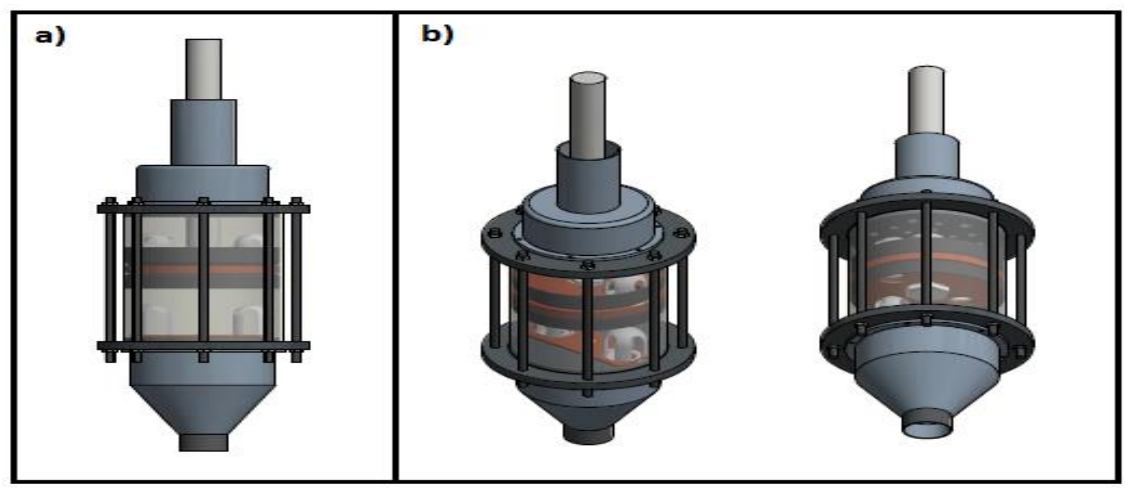

Fuente: Autores, 2019 


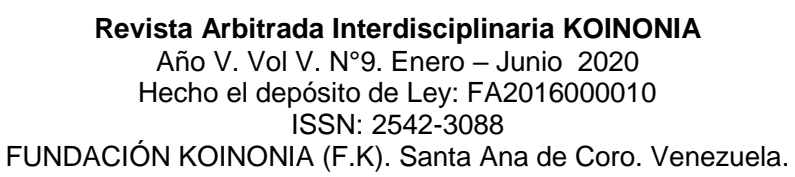

Byron Solorzano-Castillo; Jairo Castillo-Calderón; Renato Ambuludí-Hualpa

\section{REFERENCIAS CONSULTADAS}

Marrero, S. (2009). Diseño Paramétrico Basado en Modelos Matemáticos. Caso de estudio: Máquinas para la Construcción Sostenible de Viviendas (Tesis doctoral). [Parametric Design based on Mathematical Models. Case study: Machines for the Sustainable Construction of Houses (Doctoral thesis)]. Facultad de Ingeniería Mecánica. Instituto Superior Politécnico José Antonio Echeverría,Cuba.

Wielinga, B., Akkermans, J. \& Schreiber, G. (1995). A Formal Analysis of Parametric Design Problem Solving. [Un análisis formal de la resolución de problemas de diseño paramétrico]. In Proceedings 9th International Knowledge Acquisition Workshop KAW'95, 37.1-37.15.

Marrero, S. y Martínez, J. (2008). Estrategia para el Diseño Paramétrico Basado en Modelos. Ingeniería Mecánica. [Strategy for Parametric Design Based on Models. Mechanical Engineering]. Volumen (3), 39-46. Recuperado de https://www.redalyc.org/pdf/2251/225115162006.pdf

Pineda, W. (2014). Diseño y construcción de una bomba de pistón alternativa sumergible para el molino multipala americano del AARNR de la UNL (Tesis de pregrado). [Design and construction of a submersible alternative piston pump for the American multi-blade mill of the UNL AARNR (Undergraduate thesis)]. Universidad Nacional de Loja,Ecuador.

DNBrida. (2019). Bridas para tubería y accesorios bridados de NPS 1/2" a NPS 24" Métrico/Pulgadas Standard. [Pipe Flanges and Flanged Fittings from NPS $1 / 2$ "to NPS 24" Metric / Standard Inches]. Barcelona, España. Recuperado dehttp://www.dnbrida.com/brida-asme-b16.5.php.

Budynas, R; Keith, J. (2008). Diseño en Ingeniería Mecánica de Shigley. [Shigley Mechanical Engineering Design]. México: McGraw-Hill Interamericana.

Solórzano, B. (2014). Construcción de un modelo matemático integral de un compresor de tipo reciprocante, de pistón, mono-cilíndrico, vertical y de simple acción, destinado a procesos especiales (Tesis de maestría). [Construction of a comprehensive mathematical model of a single-cylinder, vertical, single-cylinder, reciprocating, piston-type compressor for special processes (Master's thesis)]. Facultad de Ingeniería Mecánica. Instituto Superior Politécnico José Antonio Echeverría,Cuba. 
Byron Solorzano-Castillo; Jairo Castillo-Calderón; Renato Ambuludí-Hualpa

Hamrock, B; Jacobson, B; Schmid, S. (2000). Elementos de Máquinas. [Machine Elements]. México: McGraw-Hill Interamericana.

Myzka, D. (2012). Máquinas y Mecanismos. [Machines and Mechanisms]. Pearson Education.

Mott, R; Untener, J. (2015). Mecánica de Fluidos. [Fluid mechanics]. México: Pearson.

Mataix, C. (1986). Mecánica de fluidos y máquinas hidráulicas. [Mecánica de fluidos y máquinas hidráulicas]. España: Ediciones del Castillo, S.A.

Cengel, Y; Cimbala, J. (2006). Mecánica de fluidos: fundamentos y aplicaciones. [Fluid mechanics: fundamentals and applications]. México:McGraw-Hilllnteramericana.

Hibbeler, R. (2011). Mecánica de materiales. [Materials mechanics]. México. Pearson Education.

Rodríguez, R.., Marrero, S. y Lazo, M. (2012). Aplicación de un algoritmo de reducción de grafos al Método de los Grafos Dicromáticos. [Application of a graph reduction algorithm to the Dichromatic Graph Method]. Ingeniería Mecánica, volumen (15),158-168.

Escalona, Y., Romero, A., Marrero, S. y Rodríguez, R. (2013). Sistema para la solución de problemas de cómputo basado en el Método de los Grafos Dicromáticos. [System for solving computer problems based on the Dichromatic Graph Method]. Revista Internacional de Gestión del Conocimiento y la Tecnología, volumen (1),37-46. 